\title{
Performance Comparison of Sectorization of DCT and DCT Wavelet Transformed Images in CBIR
}

\author{
Dr. H.B.Kekre \\ Sr. Professor, Computer Engineering \\ MPSTME, SVKM'S NMIMS \\ (Deemed-to be University) \\ Vile Parle West, Mumbai-56, INDIA
}

\author{
Dhirendra Mishra \\ Associate Professor and PhD Research Scholar \\ MPSTME, SVKM'S NMIMS \\ (Deemed-to be University) \\ Vile Parle West, Mumbai-56, INDIA
}

\begin{abstract}
This paper mainly discusses the use of discrete cosine transform and its wavelet for feature extraction in the application of content based image retrieval. The proposed method has been experimented based on the concept of sectorization of the transformed images using DCT and DCT Wavelet. For the detailed study of the behavior of the method the sector sizes are varied in 4,8,12 and 16 sector sizes. For each sector sizes two separate similarity measures namely Euclidean distance (ED) and the sum of absolute difference (AD) are considered. So the performance of the proposed methods are compared and analyzed based on the sector sizes and type of similarity measures used. The various parameters like precision, recall and its cross over point plots, LIRS and LSRR are used to measure the overall as well as the class wise performances. The sizes of the image database consist of 1055 images distributed among 12 different classes. Comparing the performance of DCT and DCT Wavelet on the broader term it has been observed that 4 sectors and 8 sectors of DCT (column wise) and DCT Wavelet (column wise) with sum of Absolute difference as similarity measure provide the best outcome of the retrieval i.e. close to $45 \%$.
\end{abstract}

\section{General Terms}

CBIR, Image retrieval, pattern recognition

\section{Keywords}

CBIR, Precision, Recall, LSRR, LIRS, Euclidean distance, sum of absolute difference.

\section{INTRODUCTION}

The innovations taking place in the digital world has evolved itself to very large extent. The result of which has caused the maximum dependency on the computer. All the information being in any form i.e. multimedia, documents, images etc. every thing has got the right place in the computer. The computer has been proved to be the very powerful mechanism to use these digital data, storage them securely and access it efficiently whenever required. Digital Images play a very important role for describing the detailed information about man, money, machine etc. almost in every field. The various processes of digitizing the images to obtain it in the best quality for the more clear and accurate information leads to the requirement of more storage space and better storage and accessing mechanism in the form of hardware or software. As far as the accessing of these images are concerned one needs to have the good mechanism of not only for accessing of the images but also for any other image processing to be done one needs to have the faster, accurate, efficient retrievals of these images. There are various approaches of proposing the methodologies of retrieving the images from the large databases consisting of millions of images stored.

Content based image retrieval (CBIR) [1-4] is one of the evolving approaches which has been researched upon to use content of the image itself to draw out its uniqueness. This uniqueness can make one to differentiate the images with each other which can provide the retrieval of the relevant images as per the requirement. There are mainly three attributes i.e. shape, color and textures of the image as of now is being experimented by many researchers. These attributes leads one to extract the exact feature of the image which can be well utilized to compare with all images available in the database by means of some similarity measures like Euclidean distance, sum of absolute difference so on. The tremendous use of images in the digital world of today has proved the CBIR as very useful in various applications like Finger print recognition, Iris Recognition, Face recognition, Palm print recognition, Speaker identification, Pattern matching and recognition etc.

There are various approaches which have been experimented to generate the efficient algorithm for CBIR. The approaches proposed by various researchers are based on both spatial domain and frequency domain. These approaches advocate different ways of extracting features of the images to improve the result in the form of better match of the query image in the large database. Some papers discuss the variation in the similarity measures in order to have its lesser complexity and better match [5-15]. Methods of feature extraction using Vector Quantization [16], Bit Truncation Coding [17,18], Walsh Transform[20,21] has also provided the new horizon to the feature extraction methodology. The method of sectorization has already been experimented on DCT [22], DST [23], DCT-DST Plane [24], Haar Wavelet [25] and Kekre's Transform [26] earlier. This paper we propose new transform named as DCT Wavelet generated from DCT matrix. The use of sectorization of DCT Wavelet for feature extraction in CBIR has been discussed. The outcome of which has been compared with the DCT sectorization performance.

The section 2 of this paper focuses on the concept of Wavelet formation from the discrete cosine transform and its sectorization. 
The section 3 discusses the sectorization of the transformed images and extraction of features to generate the feature database. It also focuses on the basic definition of row wise, column wise and full transformations. The algorithmic performance measuring parameters are discussed.

Section 4 organizes the discussion of the results plotted in the form of class wise average precision -recall cross over point, Length of initial relevant string (LIRS) and Length of string to recover all relevant images (LSRR) for all approaches. Finally concludes with overall comparison of DCT and DCT Wavelet.

Finally the paper discusses the conclusion of the outcome for the methodologies proposed

\section{DCT AND DCT WAVELET}

\subsection{Discrete cosine Transform [22]}

DCT is formed consisting of cosine functions considered over half the interval with division of this half interval into $\mathrm{N}$ equal parts. Sampling each function at the center of these parts gives orthogonal sequences. The DCT matrix is formed by arranging these sequences row wise. The DCT definition of 1 dimensional Sequence of Length $\mathrm{N}$ is given as [22]

$$
C(u)=\alpha(u) \sum_{x=0}^{N-1} f(x) \cos \left[\frac{\pi(2 x+1) u}{2 N}\right],
$$

For $\mathrm{u}=0,1,2 \ldots \mathrm{N}-1$

Similary inverse transform is given as :

$$
f(x)=\sum_{u=0}^{N-1} \alpha(u) C(u) \cos \left[\frac{\pi(2 x+1) u}{2 N}\right],
$$

For $\mathrm{x}=0,1,2 \ldots \mathrm{N}-1$ and $\alpha(\mathrm{u})$ for both equations (1) and (2) is defined as :

$$
\alpha(u)=\left\{\begin{array}{lll}
\sqrt{\frac{1}{N}} & \text { for } & u=0 \\
\sqrt{\frac{2}{N}} & \text { for } & u \neq 0 .
\end{array}\right.
$$

\subsection{DCT Wavelet Generation [4]}

The wavelet analysis procedure is to adopt a wavelet prototype function, called an analyzing wave or mother wave. Other wavelets are produced by translation and contraction of the mother wave. By contraction and translation infinite set of functions can be generated. This set of functions must be orthogonal and this condition qualifies a transform to be a wavelet transform. Thus there are only few functions which satisfy this condition of orthogonality. Generation of DCT Wavelet transform matrix of size $\mathrm{N}^{2} \mathrm{xN}^{2}$ from DCT matrix of size $\mathrm{NxN}$ is given in [4].However in this case we require DCT matrix of size $128 \times 128$ where 128 is not a square. To simplify this situation, this paper proposes an algorithm to generate discrete cosine wav elet transform from discrete cosine transform of $8 \times 8$ and $16 \times 16$.

In this paper the DCT Wavelet has been generated using the contraction and translation. Due to the size of images in the database is $128 \times 128$ we need the wavelet transform to be of size $128 \times 128$. The $128 \times 128$ Wavelet transform matrix generated from 16x16 orthogonal DCT matrix and 8x8 DCT matrix. First 16 rows of Wavelet transform matrix are generated by repeating every column of DCT matrix of dimension $16 \times 16,8$ times. To generate next 17 to 32 rows, second row of DCT (8X8) is translated using groups of 8 columns with horizontal and downward shifts. To generate next 33 to 48 rows, third row of DCT (8X8) matrix is used in the same manner. Like wise to generate last 113 to 128 rows, 8 th row of transform DCT $(8 \times 8)$ matrix is used. Note that by repeating every column of the basic transform 8 times we get global components. Other wavelets are generated by using rows of DCT matrix of size 8 x8 giving local components of the DCT Wavelet.

\section{SECTORIZATION AND FEATURE EXTRACTION}

Once the DCT Wavelet has been generated, Images in the database and query images are transformed using row wise, column wise and full transformation for both DCT and DCT Wavelet. The figure given below shows the formal steps of feature vector generation in brief.

\section{Images are transdormed using DCT and DCT Wavelet seperately}

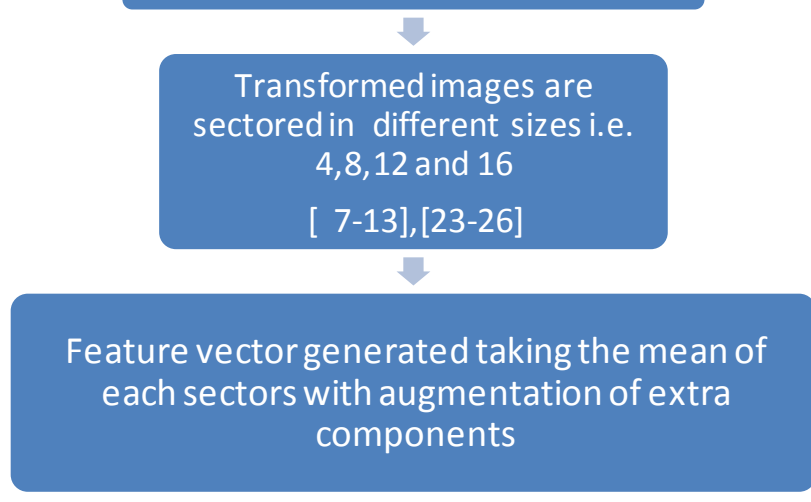

Figure $1 \mathrm{~S}$ teps to generate the feature vectors

\subsection{Row wise Transformation [24]}

The row wise transformation of DCT and DCT Wavelet 
[DCT_ WT] is given by :

$\mathrm{F}=\mathrm{f} *[\mathrm{DCT}]^{\mathrm{T}}$

$F=f^{*}\left[D C T \_W T\right]^{T}$

Where $\mathrm{F}$ is transformed image, $\mathrm{f}$ is input image to be transformed

\subsection{Column wise Transformation [27]}

The column wise transformation of DCT and DCT Wavelet [DCT_WT] is given by :

$\mathrm{F}=[\mathrm{DCT}] * \mathrm{f}$

$\mathrm{F}=\left[\mathrm{DCT} \_\mathrm{WT}\right] * \mathrm{f}$

Where $\mathrm{F}$ is transformed image, $\mathrm{f}$ is input image to be transformed

\subsection{Full Transformation [28]}

The row wise transformation of DCT and DCT Wavelet [DCT WT] is given by:

$\mathrm{F}=[\mathrm{DCT}]^{*} \mathrm{f} *[\mathrm{DCT}]^{\mathrm{T}}$

$\mathrm{F}=\left[\mathrm{DCT} \_\mathrm{WT}\right]^{*} \mathrm{f} *\left[\mathrm{DCT} \_\mathrm{WT}\right]^{\mathrm{T}}$

Where $\mathrm{F}$ is transformed image, $\mathrm{f}$ is input image to be transformed

In the full transformation methods two complex planes have been conceptualized [28]. These two planes are sectored in the various sector sizes for the experiment. These two planes have been formed by ignoring first and last rows and columns. Remaining elements have been partitioned in a group of size $2 \times 2$.The two complex planes, plane1 and plane2, are formed by taking combination of all the elements $\mathrm{X}(\mathrm{i}, \mathrm{j}), \mathrm{Y}(\mathrm{i}+1, \mathrm{j}+1)$ and $\mathrm{X}(\mathrm{i}+1, \mathrm{j}), \mathrm{Y}(\mathrm{i}, \mathrm{j}+1)$ for $\mathrm{i}, \mathrm{j}=2,3,4, \ldots, \mathrm{N}-1$ as shown below.

\begin{tabular}{|ll|}
\hline$X(i, j)$ & $Y(i, j+1)$ \\
$X(i+1, j)$ & $Y(i+1, j+1)$ \\
\hline
\end{tabular}

\subsection{Sectorization [7-13][23-28]}

The individual components of row and column wise transformed images are distributed into different co-ordinates of Cartesian coordinate system according to their sign chan ge with 45 degree of angle of division to form four sectors. The division of each of these 4 sectors into 2 partitions forms the 8 sectors which distributed the transformed image components into its appropriate sectors. Continuing the same division concept further with change in the angle size the sector sizes of 12 and each sectors of 8 sectors are individually divided into two to obtain 16 sectors. For each individual sector sizes the mean of each sectors are taken as the feature vector component. The feature vector components of each plane i.e. R, G and B has been calculated and concatenated together with the average of first row/column and last row/column to form the final feature vector

Once features of the all images in the database are extracted using the procedure as explained the feature database is generated. The query image to be retrieved from the database has been transformed using DCT/DCT Wavelet and the extracted feature of the query image is compared with the feature database using two similarity measures i.e. Eucledian distance (ED) and sum of absolute difference (AD) as given in the equation (4) and (5) shown below :

$$
\begin{aligned}
& E D(P, Q)=\sum_{i=1}^{N}(P i-Q i)^{2} \\
& A D(P, Q)=\sum_{i=1}^{N}|P i-Q i|
\end{aligned}
$$

The match of query image feature with the feature database with the minimum value of ED/AD gives the perfect match.

The performance measure of the algorithms proposed is done with the calculation of the precision-recall and LIRS (Length of initial relev ant string of retrieval) and LSRR (Length of string to recover all relevant images in the database) refer equations (6) to (9).

$$
\begin{aligned}
& \text { Precision }=\frac{\text { Number of relevant images retrieved }}{\text { Total Number of images retrieved }} \\
& \text { Recall }=\frac{\text { Number of relevant images retrieved }}{\text { Total number of relevant images in database }} \\
& L I R S=\frac{\text { Length of initial relevant string of images }}{\text { Total relevant images in the Database }} \\
& L S R R=\frac{\text { Length of string to recover all relevant images }}{\text { Total images in the Database }}
\end{aligned}
$$

The performance of the proposed methods are checked by means of calculating the class wise average performance and overall average performance of each approach with respect to the transformation method applied, the way of applying the transformation i.e. row wise, column wise, full, sector sizes used and type of similarity measures used.

\section{RESULTS AND DISCUSSION 4.1 Image Database}

The sample Images of the augmented Wang database [29] consists of 1055 images having 12 different classes such as Cartoons, Flowers, Elephants, Barbie, Mountains, Horses, 
Buses, Sunset, Tribal, Beaches, Monuments and Dinosaur shown in the Figure 2.

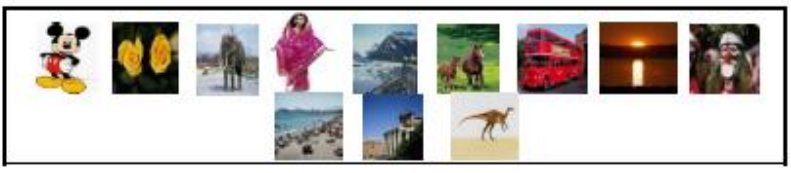

Figure 2. Sample images in the Database

The class wise distribution of all images in its respective classes such as ther are 46 images of cartoon in the class, there are 100 images for flower and so on are shown in the Figure 3 below.

\begin{tabular}{|l|l|l|l|l|l|}
\hline $\begin{array}{l}\text { Cartoon } \\
46\end{array}$ & $\begin{array}{l}\text { Flower } \\
100\end{array}$ & $\begin{array}{l}\text { Elephants } \\
100\end{array}$ & $\begin{array}{l}\text { Barbie } \\
59\end{array}$ & $\begin{array}{l}\text { Mountains } \\
100\end{array}$ & $\begin{array}{l}\text { Horse } \\
100\end{array}$ \\
& & $\begin{array}{l}\text { Monu } \\
\text { ments } \\
100\end{array}$ & $\begin{array}{l}\text { Dinasaour } \\
100\end{array}$ & \\
\hline $\begin{array}{l}\text { Sunset } \\
51\end{array}$ & $\begin{array}{l}\text { Tribal } \\
100\end{array}$ & $\begin{array}{l}\text { Beaches } \\
99\end{array}$ & & \\
\hline
\end{tabular}

Figure 3. Class wise distribution of images

\subsection{Sectorization of Row wise Transformed (DCT) images}

This section mainly discusses the algorithmic performance of the sectorization of row wise DCT transformed images in CBIR. The class wise precision-recall cross over point plot shown in the Figure 4 depicts the variation in the performance in all class of images. It can be very clearly seen that the sector 12 of most of the classes has good retrieval rate with maximum of $80 \%$ for dinosaur; for flowers it's more that $60 \%$ and $50 \%$ for horse, sunset and elephant class (see Figure 4). LIRS performance checks number of relevant images retrieved as he part of initial string of retrievals before getting the first irrelevant. So the maximum of LIRS can show the best retrieval performance. At the same time the LSRR performance checks the total length of retrievals carrying all relevant images. So the minimum LSRR shows good performance. The dinosaur class shows the ideal performance As shown in the Figure 5 and Figure 6.It varies for rest of the classes.

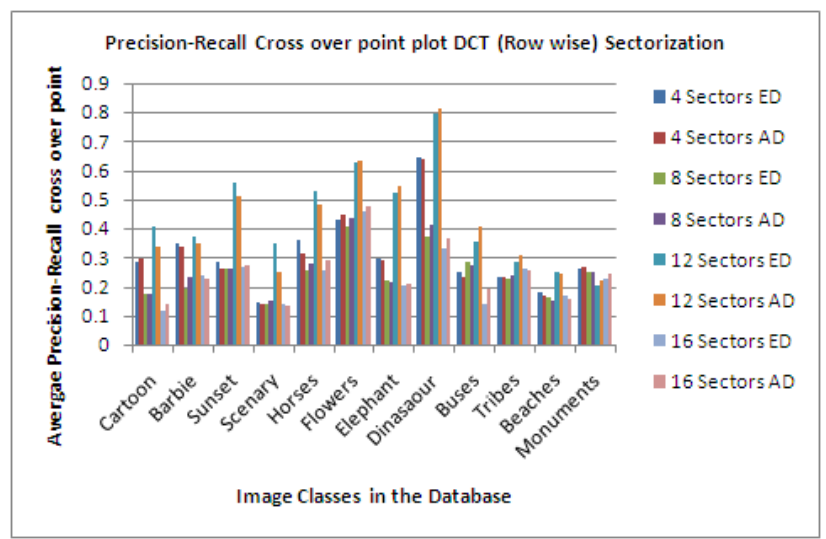

Figure4. Class wise Average Precision-Recall cross over point plot of DCT row wise sectorization for all sector sizes with respect to similarity measures i.e. Euclidian distance (ED) and sum of absolute difference (AD)

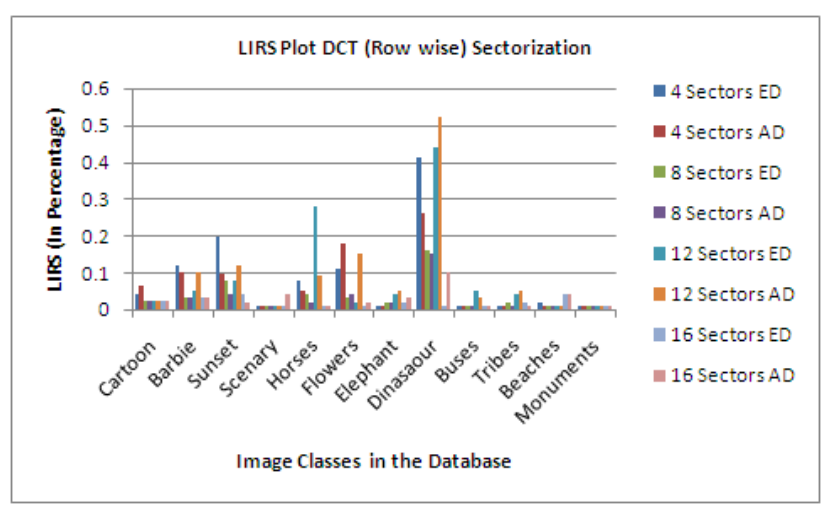

Figure5. Class wise LIRS plot of DCT row wise sectorization for all sector sizes with respect to similarity measures i.e. Euclidian distance (ED) and sum of absolute difference (AD)

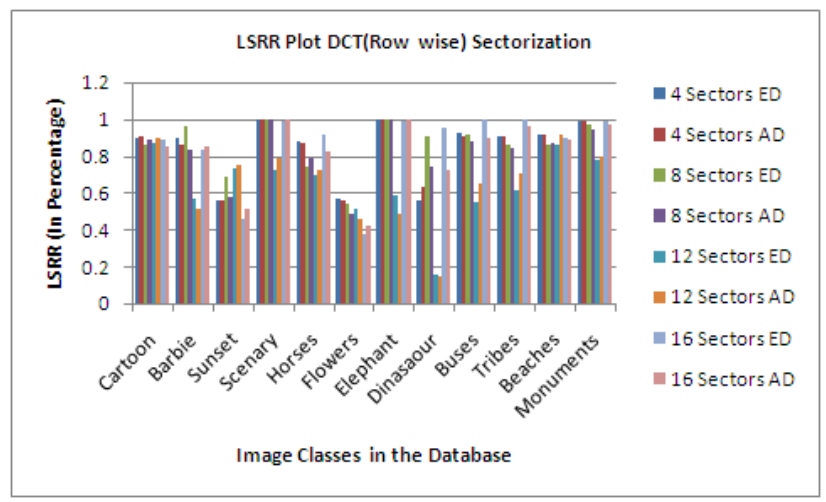

Figure6. Class wise LS RR plot of DCT row wise sectorization for all sector sizes with respect to similarity measures i.e. Euclidian distance (ED) and sum of absolute difference (AD) 


\subsection{Sectorization of Column wise Transforme d (DCT) images}

Sectorization of the column wise DCT transformed images provide the better performance of precision -Recall cross over point compared to the row wise transformation. It it shown in the Figure 7 as for the dinosaur class the sector sizes 4,8 and 16 reaches up to $80 \%$ of retrieval. The sectors 4,8 with $\mathrm{AD}$ gives $70 \%$ of retrieval for flower class whereas sector 16 with $\mathrm{AD}$ of the same class gives the retrieval more than $75 \%$.Sector 12 as not performed well for most of the classes apart from Barbie class which has up to $70 \%$ retrieval rate with ED (see the Figure 7).on average performance with respect to LIRS plot is up to $20 \%$ with the maximum of $30 \%$ for horse, Dinosaur class. LSRR plots shown in the Figure 9 shows the better performance for dinosaur, flower classes and it varies from sector to sector.

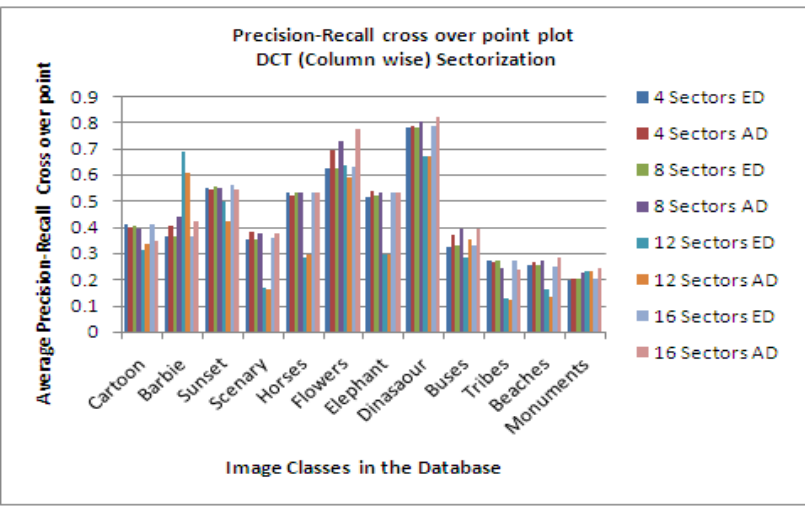

Figure7. Class wise Average Precision-Recall cross over point plot of DCT column wise sectorization for all sector sizes with respect to similarity measures i.e. Euclidian distance (ED) and sum of absolute difference (AD)

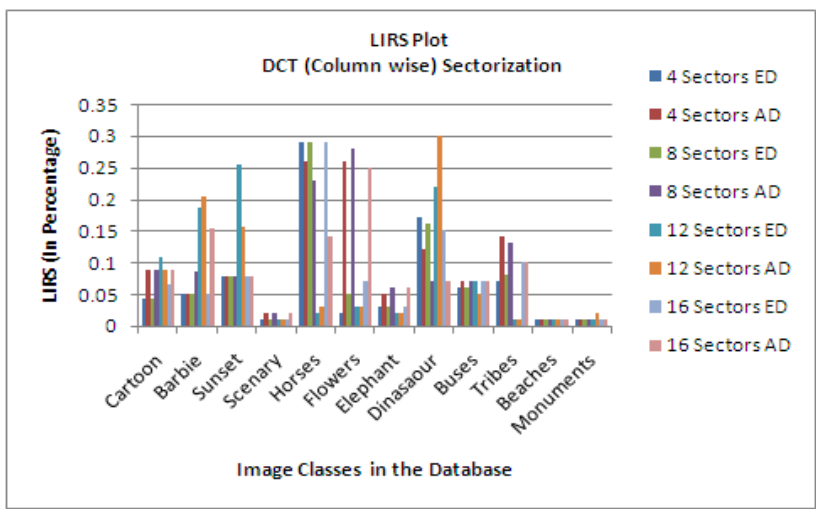

Figure 8. Class wise LIRS plot of DCT column wise sectorization for all sector sizes with respect to similarity measures i.e. Euclidian distance (ED) and sum of absolute difference (AD)

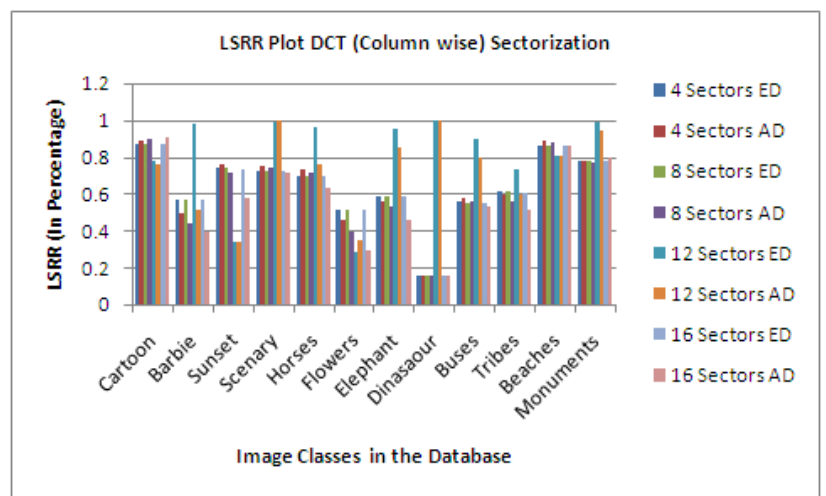

Figure9. Class wise LSRR plot of DCT column wise sectorization for all sector sizes with respect to similarity measures i.e. Eucledian distance (ED) and sum of absolute difference (AD)

\subsection{Sectorization of Full Transformed (DCT) images}

In this section the performance of Full DCT sectorization with respect to two different planes i.e. Plane1 and plane 2 has been plotted in the form of average precision-recall cross over point in Figure 10; LIRS plot in Figure 11 and LSRR plot in Figure 12. According to the precision-recall cross over point The best retrieval is again for dinosaur class given by all sectors with AD as similarity measure which makes the count to reach to $80 \%$.The second ranked retrieval outcome is for flower class well above $78 \%$ sector $8,12,16$ with AD. Next comes with the Barbie class which has retrieval up to $65 \%$ (sector size 8,12,16 with AD). The plane 2 plane has almost same retrieval rate as compared to plane1 with slight difference in flower, cartoon class as shown in the Figure 13-15.

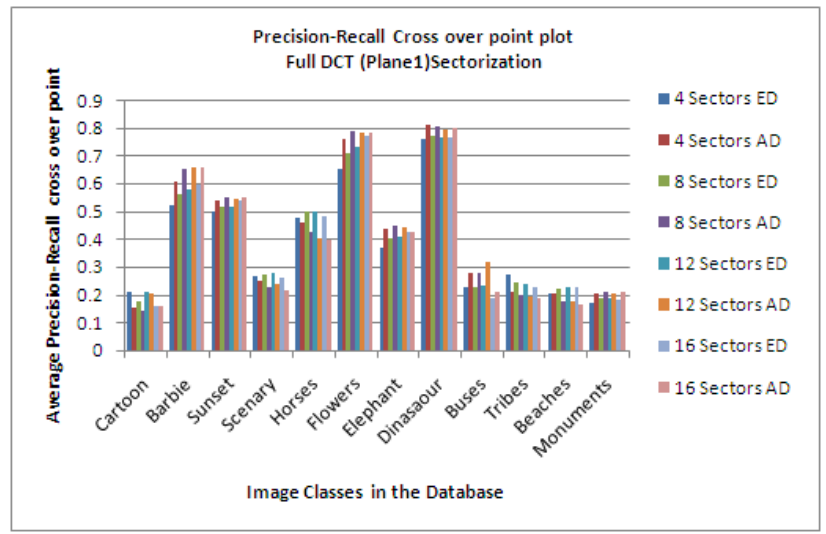

Figure 10. Class wise Average Precision-Recall cross over point plot of Full DCT (Plane1) sectorization for all sector sizes with respect to similarity measures i.e. Eucledian distance (ED) and sum of absolute difference (AD) 


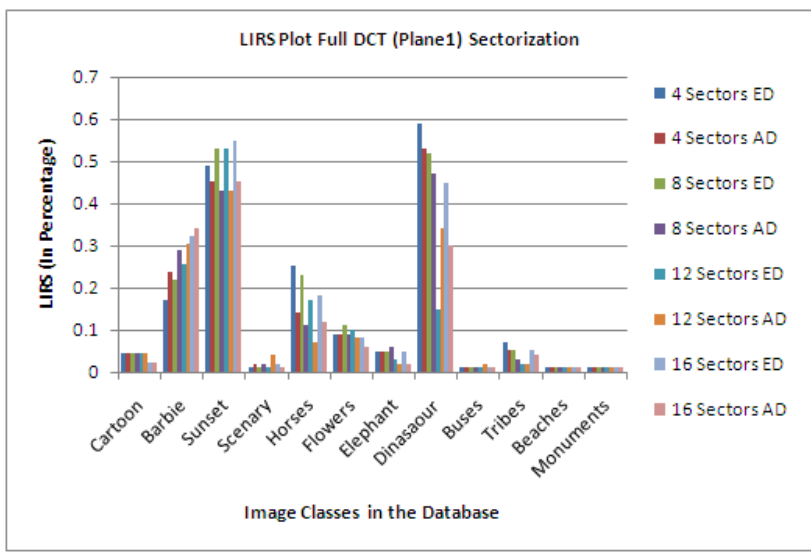

Figure 11. Class wise LIRS plot of Full DCT (Plane1) sectorization for all sector sizes with respect to similarity measures i.e. Euclidian distance (ED) and sum of absolute difference $(A D)$

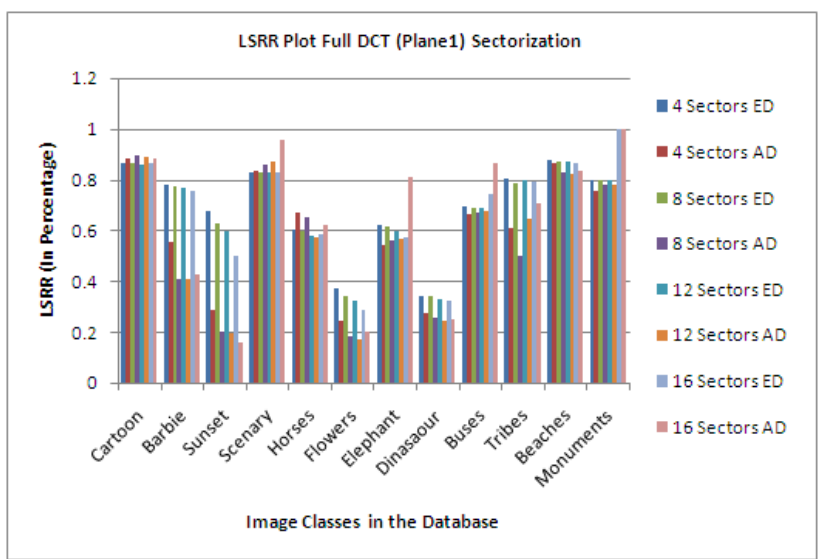

Figure 12. Class wise LS RR plot of Full DCT (Plane1) sectorization for all sector sizes with respect to similarity measures i.e. Euclidian distance (ED) and sum of absolute difference $(\mathrm{AD})$

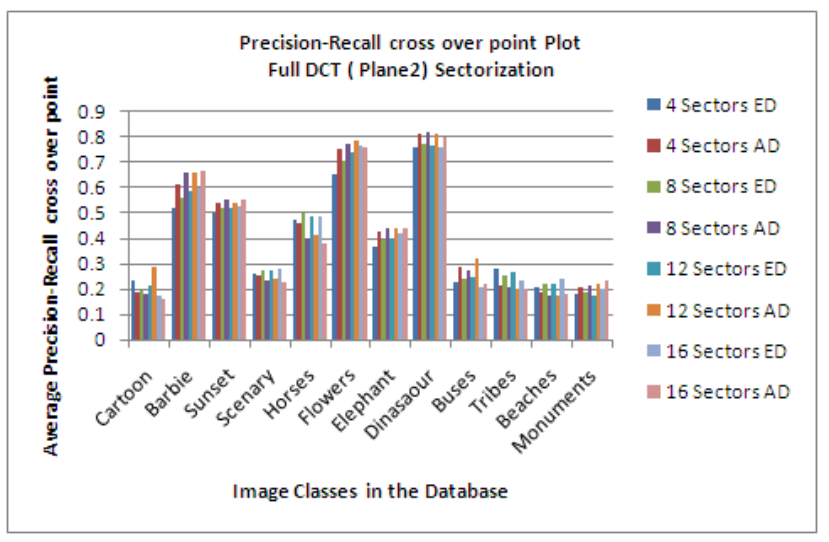

Figure 13. Class wise Average Precision-Recall cross over point plot of Full DCT (Plane2) sectorization for all sector sizes with respect to similarity measures i.e. Euclidian distance (ED) and sum of absolute difference (AD)

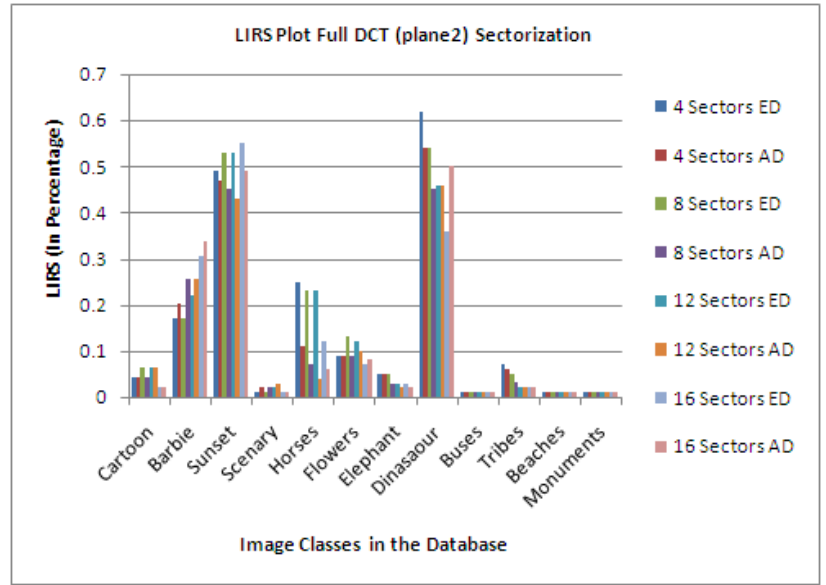

Figure 14. Class wise LIRS plot of Full DCT (Plane2) sectorization for all sector sizes with respect to similarity measures i.e. Euclidian distance (ED) and sum of absolute difference (AD)

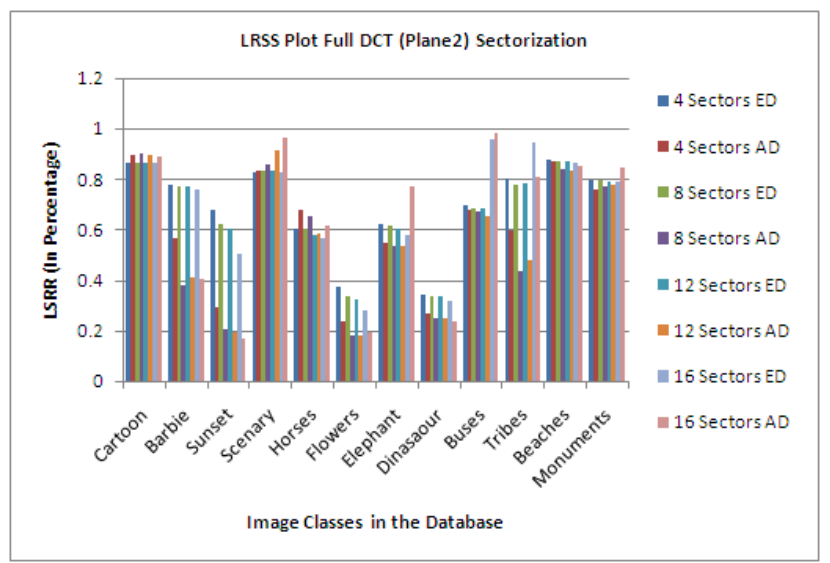

Figure 15. Class wise LSRR plot of Full DCT (Plane2) sectorization for all sector sizes with respect to similarity measures i.e. Euclidian distance (ED) and sum of absolute difference (AD)

\subsection{Overall Comparison}

This section compares the overall performance of all methods implemented i.e. DCT (row, column, full), DCT Wavelet (row, column, full) with respect to all performance measuring parameters namely precision-recall cross over point plot shown in Figure 16,the Overall average LIRS plot shown in the Figure 17 and the overall average LSRR plot in the figure 18. It is seen from the graph to comment that the column wise approach in all cases provide the better performance as compared to row wise transformation. For full transformation sectorization of both planes provide the retrieval up to $40 \%$. It is found that the all sectors of DCT Wavelet (row wise and column transformation) has retrieval performance closer to $45 \%$. 


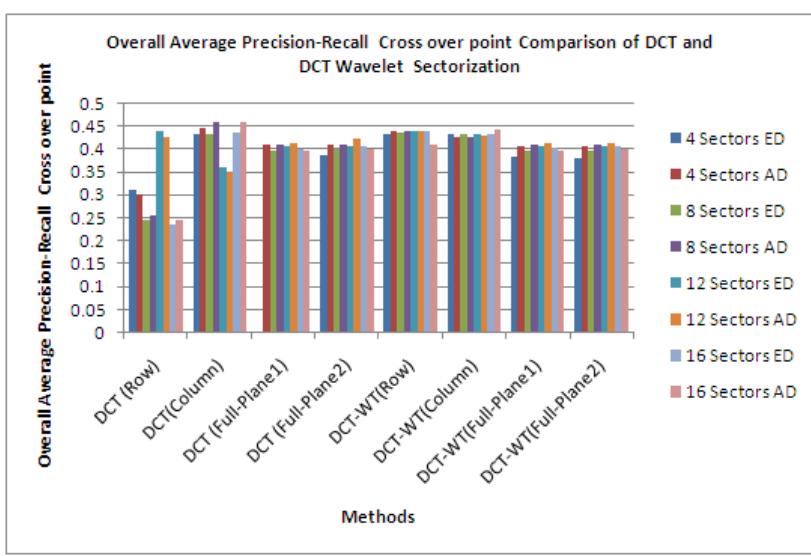

Figure 16. Overall Average Precision-Recall cross over point plot comparison of DCT and DCT Wavelet Sectorization for all sector sizes with respect to similarity measures i.e.

Euclidian distance (ED) and sum of absolute difference (AD)

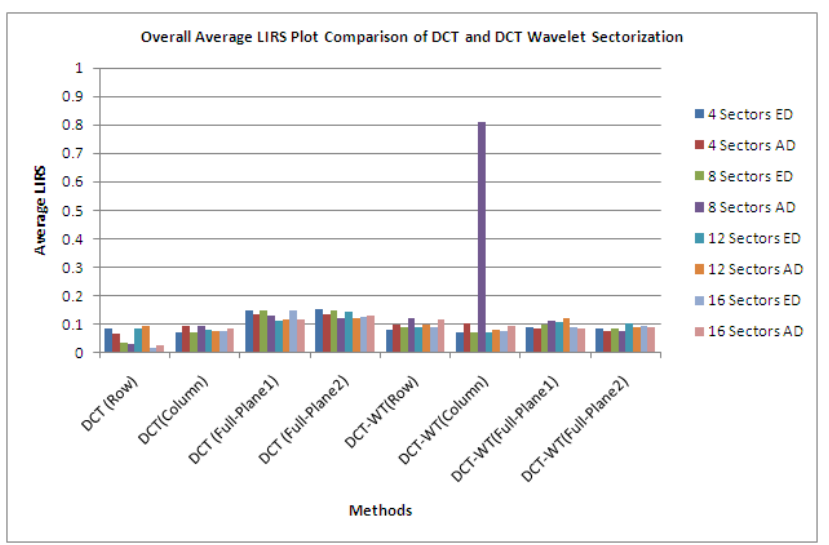

Figure 17. Overall Average LIRS plot comparison of DCT and DCT Wa velet Sectorization for all sector sizes with respect to similarity measures i.e. Euclidian distance (ED) and sum of absolute difference (AD)

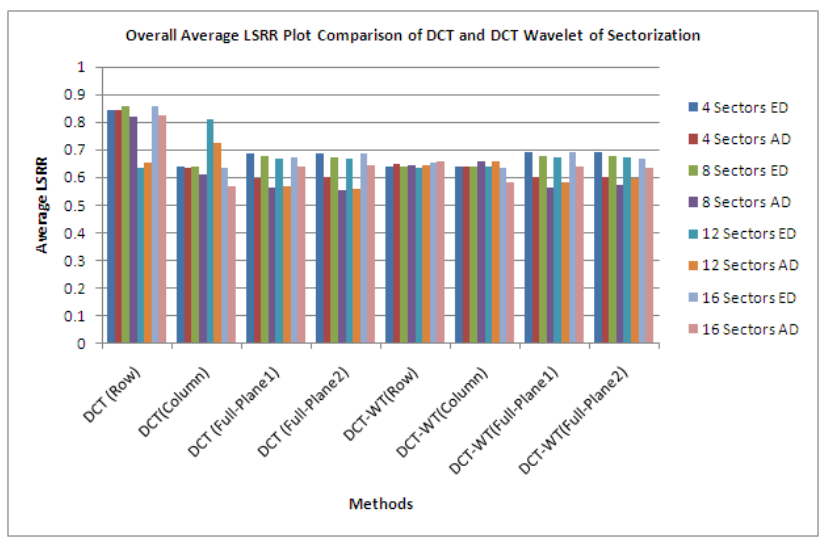

Figure 18. Overall Average LS RR plot compa rison of DCT and DCT Wavelet Sectorization for all sector sizes with respect to similarity measures i.e. Euclidian distance (ED) and sum of absolute difference (AD)

\section{CONCLUSION}

The paper compares the retrieval performance of DCT and DCT Wavelet sectorization. The performance of both of these approaches has been analyzed based on the variation in the sizes of the sectors and the type of similarity measures namely Euclidian distance (ED) and sum of absolute difference (AD) and the transformation approaches i.e. row wise, column wise and full. Two planes namely have been formed in the Full transformation. These two planes are sectored in various sizes to check its effect in the final outcome. The performance measuring parameters like precision-recall cross over point plot, LIRS Plot and LSRR Plot has been used. Comparing the performance of DCT and DCT Wavelet on the broader term it has been observed that 8 sectors and 16 sectors of DCT (column wise) with Absolute difference provide the best outcome of the retrieval i.e. more than $45 \%$.The outcome of retrieval for DCT Wavelet (row wise and column wise) is close to $45 \%$. There is variation in the results with respect to the sector sizes in these approaches. All sector sizes perform well up to $40 \%$ in the case of the DCT(column wise ) except sector 12. whereas for DCT Wavelet all sectors has good performance. All sectors with both $\mathrm{ED}$ and $\mathrm{AD}$ of DCT Wavelet (row wise) sectorization has retrieval rate more than $40 \%$ which is far better than DCT (row wise) which is only up to $30 \%$. In general column wise and full plane sectors give better performance as compared to row wise. Whereas for full transform plane1 and plane2 give similar performance. The best performance is obtained for dinasour and flowers class which is up to $80 \%$.

\section{REFERENCES}

[1] Kato, T., "Database architecture for content based image retrieval in Image Storage and Retrieval Systems" (Jambardino A and Niblack W eds),Proc SPIE 2185, pp 112-123, 1992

[2 ] Ritendra Datta,Dhiraj Joshi,Jia Li and James Z. Wang, “ Image retrieval:Idea,influences and trends of the new age",ACM Computing survey,Vol 40,No.2,Article 5,April 2008.

[3] John Berry and David A. Stoney "The history and development of fingerprinting," in Advances in Fingerprint Technology, Henry C. Lee and R. E. Gaensslen, Eds., pp. 1-40. CRC Press Florida, $2^{\text {nd }}$ edition, 2001.

[ 4 ] H.B.Kekre, Archana Athawale, Dipali sadavarti, "Algorithm to generate wavelet transform from an orthogonal transform", International journal of Image processing, Vol.4(4), pp.444-455

[ 5 ] H. B. Kekre, Dhirendra Mishra, "Digital Image Search \& Retrieval using FFT Sectors" published in proceedings of National/Asia pacific conference on Information communication and technology(NCICT 10) $5^{\mathrm{TH}} \& 6^{\mathrm{TH}}$ March 2010.SVKM'S NMIMS MUMBAI

[ 6 ] H.B.Kekre, Dhirendra Mishra, "Content Based Image Retrieval using Weighted Hamming Distance Image hash Value" published in the proceedings of 
international conference on contours of computing technology pp. 305-309 (Thinkquest2010) 13th \& $14^{\text {th }}$ March 2010.

[ 7 ] H.B.Kekre, Dhirendra Mishra,"Digital Image Search \& Retrieval using FFT Sectors of Color Images" published in International Journal of Computer Science and Engineering (IJCSE) Vol. 02,No.02,2010,pp.368-372 ISSN 0975-3397 available online

http://www.enggjournals.com/ijcse/doc/IJCSE10-0202-46.pdf

[ 8 ] H.B.Kekre, Dhirendra Mishra, "CBIR using upper six FFT Sectors of Color Images for feature vector generation" published in International Journal of Engineering and Technology(IJET) Vol. 02, No. 02, 2010, 49-54 ISSN 0975-4024 available online at http://www.enggjournals.com/ijet/doc/IJET10-0202-06.pdf

[ 9] H.B.Kekre, Dhirendra Mishra, "Four walsh transform sectors feature vectors for image retrieval from image databases", published in international journal of computer science and information technologies (IJCSIT) Vol. 1 (2) 2010, 33-37 ISSN 0975-9646 available online

at http://www.ijcsit.com/docs/vollissue2/ijcsit201001020 1.pdf

[ 10 ] H.B.Kekre, Dhirendra Mishra, "Performance comparison of four, eight and twelve Walsh transform sectors feature vectors for image retrieval from image databases", published in international journal of Engineering, science and technology(IJEST) Vol.2(5) 2010, 1370-1374 ISSN 0975-5462 available online at http://www.ijest.info/docs/IJEST10-02-05-62.pdf

[ 11 ] H.B.Kekre, Dhirendra Mishra, "density distribution in walsh transfom sectors ass feature vectors for image retrieval", published in international journal of compute applications (IJCA) Vol.4(6) 2010, 30-36 ISSN 0975-8887 available online at http://www.ijcaonline.org/archives/volume4/number6/ 829-1072

[12] H.B.Kekre, Dhirendra Mishra, "Performance comparison of density distribution and sector mean in Walsh transform sectors as feature vectors for image retrieval", published in international journal of Image Processing (IJIP) Vol.4(3) 2010, ISSN 1985-2304 available online at http://www.cscjournals.org/csc/manuscript/Journals/IJI P/Volume4/Issue3/IJIP-193.pdf

[ 13 ] H.B.Kekre, Dhirendra Mishra, "Density distribution and sector mean with zero-sal and highest-cal components in Walsh transform sectors as feature vectors for image retrieval", published in international journal of Computer scienece and information security (IJCSIS) Vol.8(4) 2010, ISSN 1947-5500 available online http://sites.google.com/site/ijcsis/vol-8-no-4jul-2010

[14] Arun Ross, Anil Jain, James Reisman, “A hybrid fingerprint matcher," Int'l conference on Pattern Recognition (ICPR), Aug 2002.

[15] A. M. Bazen, G. T. B.Verwaaijen, S. H. Gerez, L. P. J. Veelenturf, and B. J. van der Zwaag, "A correlationbased fingerprint verification system," Proceedings of the ProRISC2000 Workshop on Circuits, Systems and Signal Processing, Veldhoven, Netherlands, Nov 2000.

[ 16] H.B.Kekre, Tanuja K. Sarode, Sudeep D. Thepade, "Image Retrieval using Color-Texture Features from DCT on VQ Codevectors obtained by Kekre's Fast Codebook Generation", ICGST International Journal on Graphics, Vision and Image Processing (GVIP), Available online at http://www.icgst.com/gvip

[ 17 ] H.B.Kekre, Sudeep D. Thepade, "Using YUV Color Space to Hoist the Performance of Block Truncation Coding for Image Retrieval”, IEEE International Advanced Computing Conference 2009 (IACC'09), Thapar University, Patiala, INDIA, 6-7 M arch 2009.

[18] H.B.Kekre, Sudeep D. Thepade, "Image Retrieval using Augmented Block Truncation Coding Techniques", ACM International Conference on Advances in Computing, Communication and Control (ICAC3-2009), pp.: 384-390, 23-24 Jan 2009, Fr. Conceicao Rodrigous College of Engg., Mumbai. Available online at ACM portal.

[ 19] H.B.Kekre, Tanuja K. Sarode, Sudeep D. Thepade, "DCT Applied to Column mean and Row Mean Vectors of Image for Fingerprint Identification", International Conference on Computer Networks and Security, ICCNS-2008, 27-28 Sept 2008, Vishwakarma Institute of Technology, Pune.

[20] H.B.Kekre, Sudeep Thepade, Archana Athawale, Anant Shah, Prathmesh Velekar, Suraj Shirke, "Walsh transform over row mean column mean using image fragmentation and energy compaction for image retrieval", International journal of computer science and en gineering (IJCSE), Vol.2.No.1,S2010,47-54.

[ 21 ] H.B.Kekre, Vinayak Bharadi, "Walsh Coefficients of the Horizontal \& Vertical Pixel Distribution of Signature Template", In Proc. of Int. Conference ICIP07, Bangalore University, Bangalore. 10-12 Aug 2007.

[22 ] H.B.Kekre, Dhirendra Mishra, "DCT Sectorization for feature vector generation in CBIR", International journal of computer Applications (IJCA),Vol.9,No.1,pp.19-26

[ 23 ] H.B.Kekre, Dhirendra Mishra, "DST Sectorization for Feature vector generation", Universal journal of computer science and and Engineering Technology (UniCSE), Vol.1, No.1, Oct.2010,pp.6-15,Available online http://www.unicse.org/index.php?option=com_content $\&$ view $=$ article $\&$ id $=54 \&$ Itemid $=27$

[ 24 ] H.B.Kekre, Dhirendra Mishra, "DCT-DST Plane sectorization of row wise transformed color images in CBIR", International journal of engineering science 
and technology, Vol.2, No.12, Dec.2010, pp.72347244, ISSN No.0975-5462. Available at http://www.ijest.info/docs/IJEST10-02-12-143.pdf

[ 25 ] H.B.Kekre, Dhirendra Mishra, "Sectorization of Haar and Kekre's Wavelet for feature extraction of color images in image retrieval", International journal of computer science and information security (IJCSIS), USA, Vol.9, No.2, Feb 2011, pp.180-188, http://sites.google.com/site/ijcsis/volume-9-no-2-feb2011

[26] H.B.Kekre, Dhirendra Mishra, "Sectorization of Kekre's transform for image retrieval in content based image retrieval", Journal of Telecommunication (JOT),UK, Vol.8, No.1 April 2011, pp. 26-33. http://sites.google.com/site/journaloftelecommunicatio ns/volume-8-issue-1-april-2011

[ 27 ] H.B.Kekre, Dhirendra Mishra, "Sectorization of DCTDST Plane for column wise transformed color images in CBIR", International Conference of Technology Systems \& Management (ICTSM-2011) held at SVKM's NMIMS Mumbai India, published in Springer Link CCIS 145, pp. 55-60, 2011. Available online at

http://www.springerlink.com/content/m573256n53r077 $33 /$

[28 ] H.B.Kekre, Dhirendra Mishra, "Full DCT sectorization for Feature vector generation in CBIR", Journal of graphics, vision, image processing, Vol.11, No.2, April 2011, pp. 19 - 30 http://www.icgst.com/gvip/Volume11/Issue2/P115104 1315.html

[29] Jia Li, James Z. Wang, “Automatic linguistic indexing of pictures by a statistical modeling approach," IEEE Transactions on Pattern Analysis and Machine Intelligence, vol. 25, no. 9, pp. 1075-1088, 2003

\section{AUTHORS PROFILE}

H. B. Kekre has received B.E. (Hons.) in Telecomm. Engg. from Jabalpur University in 1958, M.Tech (Industrial Electronics) from IIT Bombay in 1960, M.S.Engg. (Electrical Engg.) from University of Ottawa in 1965 and Ph.D.(System Identification) from IIT Bombay in 1970. He has worked Over 35 years as Faculty and H.O.D. Computer science and Engg. At IIT Bombay. From last 13 years working as a professor in Dept. of Computer Engg. at Thadomal Shahani Engg. College, Mumbai. He is currently senior Professor working with Mukesh Patel School of Technology Management and Engineering, SVKM's NMIMS University vile parle west Mumbai. He has guided 17 PhD.s 150 M.E./M.Tech Projects and several B.E./B.Tech Projects. His areas of interest are Digital signal processing, Image Processing and computer networking. He has more than 350 papers in National/International Conferences/Journals to his credit. Recently twelve students working under his guidance have received the best paper awards. Two research scholars working under his guid ance have been awarded $\mathrm{Ph}$. D. degree by NMIMS University. Currently he is guiding $10 \mathrm{PhD}$. Students. He is life member of ISTE and Fellow of IETE.

Dhirendra Mishra has received his BE (Computer Engg) degree from University of Mumbai. He completed his M.E. (Computer Engg) from Thadomal shahani Engg. College, Mumbai, University of Mumbai. He is PhD Research Scholar and working as Associate Professor in Computer Engineering department of, Mukesh Patel School of Technology Management and Engineering, SVKM's NMIMS University, Mumbai, INDIA. He is life member of Indian Society of Technical education (ISTE), Member of International association of computer science and information technology (IACSIT), Singapore, Member of International association of Engineers (IAENG). He has 30 papers in National/International conferences and journals to his credit. His areas of interests are Image Processing, Operating system, Information Storage and Management 